\title{
Multi-Wavelength Ultra-Weak Fiber Bragg Grating Arrays for Long-Distance Quasi-Distributed Sensing
}

\author{
Wenjing $\mathrm{GAO}^{1,2 \dagger}$, Jianxia $\mathrm{LIU}^{1,2 \dagger}$, Huiyong $\mathrm{GUO}^{2}$, Xin JIANG ${ }^{2}$, \\ Shaofa $\mathrm{SUN}^{1}$, and Haihu $\mathrm{YU}^{2 *}$

\begin{abstract}
${ }^{1}$ Hubei Key Laboratory of Radiation Chemistry and Functional Materials, Hubei University of Science and Technology, Xianning 437100, China

${ }^{2}$ National Engineering Laboratory for Fiber Optic Sensing Technology, Wuhan University of Technology, Wuhan 430070, China
\end{abstract} \\ ${ }^{\dagger}$ These authors contributed equally \\ ${ }^{*}$ Corresponding author: Haihu YU E-mail: hhyu@whut.edu.cn
}

\begin{abstract}
Fiber Bragg grating (FBG) array, consisting of a number of sensing units in a single optical fiber, can be practically applied in quasi-distributed sensing networks. Serious signal crosstalk occurring between large-serial of identical FBGs, however, has limited the further increase in the number of sensing units, thus restricting applications only for short-distance sensing networks. To reduce the signal crosstalk, we design two novel types of 10-kilometer-long FBG arrays with 10000 equally spaced gratings, written on-line using a customized grating inscription system, which is affiliated to a drawing tower. Main factors causing signal crosstalk, such as spectral shadowing and multiple reflections, are firstly investigated in theory. Consistent with the theoretical findings, experimental results are proving that ultra-weak (the reflectivity of $\sim 40 \mathrm{~dB}$ ) and multi-wavelength gratings of a number more than 10000 can be readily identified, with satisfied low crosstalk. The maximum attenuation of grating signal and minimum signal-to-noise ratio (SNR) in a single-wavelength array are $10.69 \mathrm{~dB}$ and $5.62 \mathrm{~dB}$, respectively. As a comparison, by increasing the number of central wavelengths to three, the attenuation can be effectively reduced to $5.54 \mathrm{~dB}$ and the minimum SNR has been improved to $8.14 \mathrm{~dB}$. The current study significantly enhances the multiplexing capacity of FBG arrays and demonstrates promising potentials for establishing large-capacity quasi-distributed sensing networks.
\end{abstract}

Keywords: Multi-wavelength gratings array; large scale sensing network; crosstalk; ultra-weak grating

Citation: Wenjing GAO, Jianxia LIU, Huiyong GUO, Xin JIANG, Shaofa SUN, and Haihu YU, "Multi-Wavelength Ultra-Weak Fiber Bragg Grating Arrays for Long-Distance Quasi-Distributed Sensing," Photonic Sensors, 2022, 12(2): 185-195.

\section{Introduction}

Recently, there are increasing demands for on-line long-distance distributed sensing such as fire alarm systems in traffic tunnels [1], multi-parameter surveillance in oil/gas down-holes [2], process monitoring in chemical industry, security, and defense monitoring [3, 4]. Optical fiber sensors possess advantageous features such as high sensitivity, immunity to electromagnetic interference, and feasibility for long-distance applications. It is therefore fiber-based distributed sensors have been

Received: 18 January 2011 / Revised: 7 May 2021

(C) The Author(s) 2021. This article is published with open access at Springerlink.com

DOI: 10.1007/s13320-021-0635-4

Article type: Regular 
widely used for the aforementioned fields $[5,6]$. The most adopted schemes for long-distance fiber sensing are the Raman optical time-domain reflectometry (ROTDR), Brillouin optical time domain analysis (BOTDA), and phase-sensitive optical time-domain reflectometry (Ф-OTDR) [6-10]. Such distributed sensing systems have already demonstrated high performance in extended operation distance with improved spatial resolution. Even though the above sensing schemes have been intensively investigated, a fiber Bragg grating (FBG) array with a certain number of gratings has still proven to be a good alternative to be used for long-distance quasi-distributed sensing $[11,12]$. In such a system, each point of the grating can be deemed as a probe unit, responding rapidly to surrounding disturbance. By varying its parameters such as fiber length and the spacing between gratings and reflectivity, the FBG array system can be used for different sensing applications.

For an FBG array-based sensing system, the grating demodulation technology is crucial to determine whether or not the system can accurately respond to signals from each grating. When employing the wavelength-division multiplexing (WDM) technique, only tens of FBGs can be multiplexed in an array, due to the reason that the ratio of the bandwidth of a light source (usually less than $100 \mathrm{~nm}$ ) versus the dynamic wavelength range of a single FBG sensor (a few nanometers) can be very limited [13]. The time-division multiplexing (TDM) technique utilizes different time delays between reflected pulses to locate FBGs, and in principle, it can greatly reduce the required bandwidth by using identical gratings to achieve a better multiplexing capacity [13-15]. However, the employment of FBGs with high reflectivity in conventional TDM systems suffers from severe crosstalk, and consequently, the multiplexing capacity is reduced by contraries [16]. In recent years, a hybrid wavelength scanning time-division multiplexing (WSTDM) technique has been introduced [17, 18]. The WSTDM scheme allows the multiplexing of FBGs with reflectivity as low as $-50 \mathrm{~dB}$ so that a large capacity grating array can be realized, as demonstrated by Wang et al. [17]. Using an improved method, 1000 ultra-weak FBGs were inscribed over a 1-km-long fiber. The reflectivity of each grating is, however, not consistent with each other, fluctuating from $-37 \mathrm{~dB}$ to $-50 \mathrm{~dB}$. Moreover, the existence of fiber fusion points further weakens reflected signals, resulting in a low signal-to-noise ratio (SNR) and increased interrogation difficulty.

On-line writing of grating arrays using a drawing tower has proven to be an effective way for fabricating large-scale grating array networks [19, 20]. Using this method, the fabricated gratings possess improved homogeneity, presenting high consistency in grating reflectivity and low transmission loss. Especially, the writing of gratings over a single fiber is continuous during fiber drawing and therefore, unnecessary to re-connect fibers using splicing, as required by a traditional grating inscription procedure for a large number of gratings. Recently, we have been developing and optimizing the FBG array on-line fabrication technique and demonstrated its feasibility [21-23]. Over $10 \mathrm{~km}$ grating arrays can be written by our on-line inscription system, and these FBG arrays have proven to be suitable for long-distance quasi-distributed sensing networks. However, it is found that the introduction of a large number of gratings can cause serious signal crosstalk, which limits the multiplexing number [16].

To solve this problem, in this paper, two main factors influencing the multiplexing capacity are carefully studied in theory, based on which a method of achieving large-scale FBG arrays is proposed. Ultra-weak grating with a reflectivity of $-40 \mathrm{~dB}$ possesses low spectral shadowing and multiple-reflection effect and can be used for large-scale FBG arrays with lengths of several kilometers. The introduction of multi-wavelength gratings can further improve the multiplexing 
capacity, for example, a three-wavelength FBG array has doubled the multiplexing capacity than the use of a single-wavelength. Based on the theory, we then fabricate two 10-kilometer-long, high-quality FBG arrays multiplexed with as many as 10000 equally spaced ultra-weak gratings. The difference of grating spectra in the two arrays is analyzed for achieving suppressed crosstalk in the sensing networks. Both the two FBG arrays in the 10-kilometer-long fibers can be used as a whole. The maximum multiplexing capacity of the threewavelength is estimated to reach 20-kilometer-long when the grating number is further increased to 20000 .

\section{Signal crosstalk in large serial FBG arrays: a theory}

A large-serial FBG array is named for an FBG array with a large number of gratings, which are distributed over an optical fiber. With an increase in multiplexing number, serious signal crosstalk limits the effective number of gratings in such a system [18]. More precisely, signals reflected from downstream gratings cannot be accurately received by a grating interrogator, due to the introduction of a large number of upstream gratings. On the one hand, the upstream gratings can increase the insertion loss, which makes the signal power decrease significantly, when they reach the downstream gratings. It is also known as the spectral shadowing effect. This effect can be readily understood that an optical signal is partially reflected by the upstream gratings, and such reflections can be accumulated with an increase in the number of identical gratings. On the other hand, the noise signal which undergoes multiple reflections between the upstream gratings reaches the interrogator together with the real signal of the downstream gratings, leading to serious spectral distortion of the downstream gratings. It is named as multiple-reflection induced crosstalk, and such an effect can accumulate, similar to the spectral shadowing. Both the spectral shadowing and multiple-reflection induced crosstalk can be detrimental by lowering the SNR and increasing the interrogation difficulty. It is necessary to figure out a solution, for the practical applications of large serial FBG arrays, as discussed below.

For an identical FBGs array, assuming high-order reflections are neglectable, the returned power from the $i$ th $(i=1,2, \cdots, N)$ grating can be approximately described as [16]

$$
P_{i}(\lambda)=[1-R(\lambda)]^{2(i-1)} \cdot R(\lambda) P_{0}(\lambda) \cdot 10^{\frac{-\beta}{10} \cdot[2(i-1) L]}
$$

where $R(\lambda)$ is the reflection spectrum of the FBG at the wavelength $\lambda, P_{0}(\lambda)$ is the power of the input source, $L$ is the separation between two FBGs, and $\beta$ is the fiber transmission loss in $\mathrm{dB} / \mathrm{km}$.

For a real signal of the $i$ th FBG, the crosstalk arising from multiple reflections has the same transmission length over the same fiber. Here we only consider the first-order reflection as other higher-order weak reflections can be neglectable due to weak gratings (usually less than $-30 \mathrm{~dB}$ ), so the above equation can be approximated as below [16]:

$$
\begin{aligned}
P_{\text {multi }, i}(\lambda)= & \frac{(i-1)(i-2)}{2}[1-R(\lambda)]^{2(i-2)} \times \\
& R(\lambda)^{3} P_{0}(\lambda) \cdot 10^{\frac{-\beta}{10} \cdot[2(i-1) L]}, i \geq 3 .
\end{aligned}
$$

The SNR of the $i$ th real FBG is given as

$$
C_{i}=\frac{P_{\text {multi, } i}(\lambda)}{P_{i}(\lambda)}
$$

Since the crosstalk arising from multiple reflections cannot be directly distinguished from the signal, it can be studied by measuring ghost grating, which is also induced by multiple reflections. The ghost grating can form a non-existent FBG array which is right behind the real FBG array, and tune the reflected power. From [16], the reflected power from the $j$ th $(j=1,2, \cdots, N-1)$ ghost grating can be described by

$$
\begin{gathered}
P_{\text {ghost }, j}(\lambda)=\frac{(N-j+1)(N-j)}{2}[1-R(\lambda)]^{2(N+j-2)} \times \\
R(\lambda)^{3} P_{0}(\lambda) \cdot 10^{\frac{-\beta}{10}[2(N+j) L]}, i \geq 3
\end{gathered}
$$


where $N$ is the total number of real FBGs for the whole array.

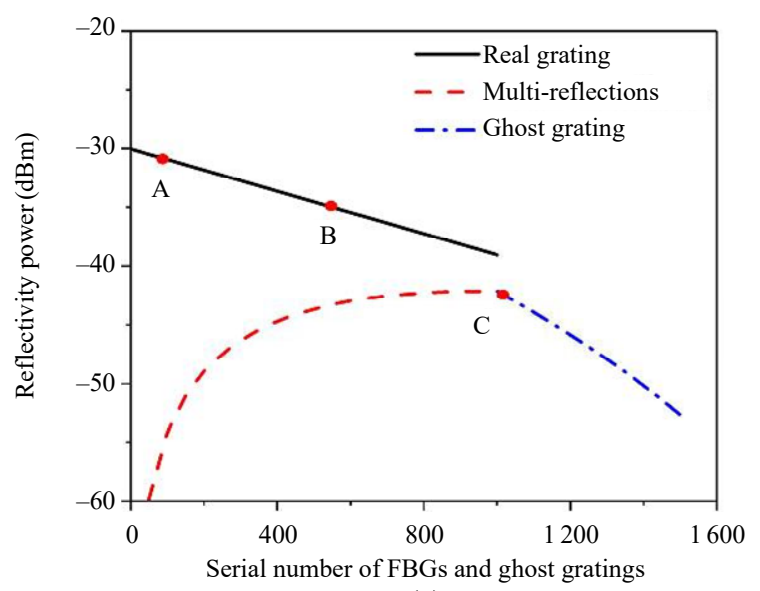

(a)

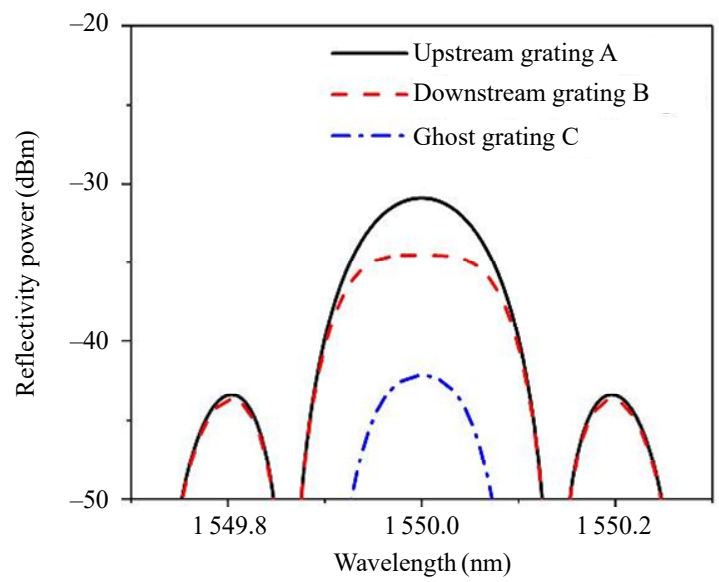

(b)

Fig. 1 Simulation of the reflected power (a) and reflection spectra (b) from the FBGs in the different locations of array.

Based on (1) - (4), we check the relationship between the signal power and the serial number of gratings. In the calculations, the power of the input source, the fiber transmission loss, and the distance of adjacent gratings are set to $0 \mathrm{dBm}, 0.2 \mathrm{~dB} / \mathrm{km}$, and $1 \mathrm{~m}$, respectively. Figure 1(a) shows the reflected power from the grating 1st to 1000 th, and the reflectivity of each grating is $-30 \mathrm{~dB}$. It mainly consists of three parts: real signal, noise from multiple reflections, and false signals from ghost gratings. As seen from Fig. 1(a), with an increase in the multiplexing number of FBGs in the array, the real signal of downstream gratings is decreased, but the noise of the corresponding grating is increased.
The ghost gratings are right behind real gratings with similar parameters but lower reflectivity. Figure 1(b) shows the reflection spectra of the upstream grating $A$ at 100th, downstream grating B at 600th, and the first ghost grating C. Due to the spectral shadowing, the reflection spectra of downstream gratings B decreases obviously, and the peak region is however flatted or even completely depressed. The ghost grating $\mathrm{C}$ has a very obvious reflection peak, and it can be regarded as the real grating by the interrogator as the number of multiplexed gratings further increases. Interestingly, the maximum value of multiple-reflection induced crosstalk in the array is consistent with the power of the first ghost grating. This is because, the maximum value of multiple-reflection induced crosstalk occurs at the end of FBG array, and it has the similar number of the first-order reflection to the 1st ghost grating "reflection". Therefore, in the following studies, we use the intensity of the first ghost grating to estimate the degree of multiplereflection induced crosstalk.

Decreasing the reflectivity of each grating has an obvious effect on attenuating the signal crosstalk. Figure 2(a) shows the relationship between power and FBG number with decreased reflectivity of a single grating to the $-34 \mathrm{~dB},-36 \mathrm{~dB},-38 \mathrm{~dB},-40 \mathrm{~dB}$, and $-42 \mathrm{~dB}$, respectively. Assuming the detection limit of an interrogator is $-50 \mathrm{~dB}$, the number of the recognized gratings can reach 4 100, 5300,6800 , 7800 , and 8500 , respectively. The relationship between SNR and the number of corresponding gratings is shown in Fig. 2(b). When the number of FBGs is small, the grating crosstalk is at a low level, which makes the signal have a high SNR. With an increase in the serial number of FBGs, the multiple-reflection induced crosstalk will increase rapidly at the beginning to make the SNR decrease quickly. After that, the real signal decreases slowly and the noise signal increases steadily to make the SNR decrease continuously. Assuming the SNR needs to be greater than $3 \mathrm{~dB}$, the number of the 
recognized grating can reach $2500,4000,6200$, 9 900, and even more than 10000 . When the reflectivity was decreased by $2 \mathrm{~dB}$, the SNR of gratings at the same locations can be increased by $4 \mathrm{~dB}$. From the above discussion, it is clear that ultra-weak FBGs (reflectivity reduced to about $-40 \mathrm{~dB})$ can be a better solution to effectively improve the multiplexing capacity, over a long fiber with a large number of FBGs.

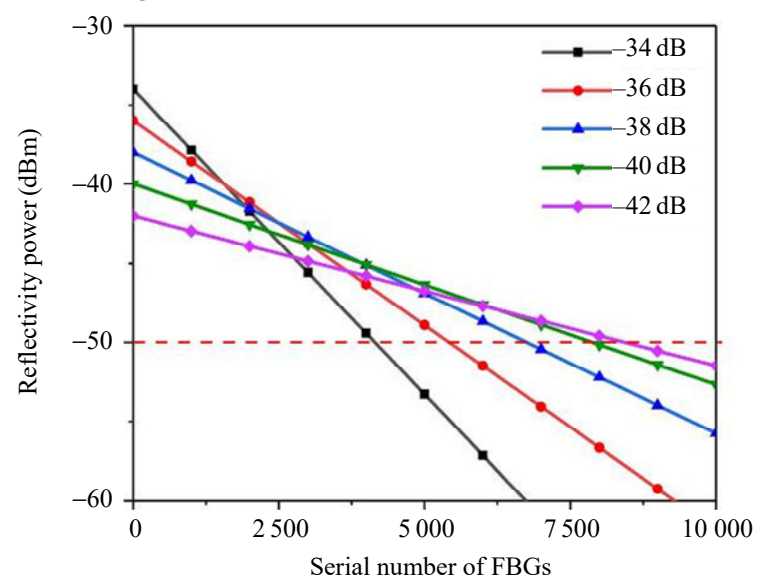

(a)

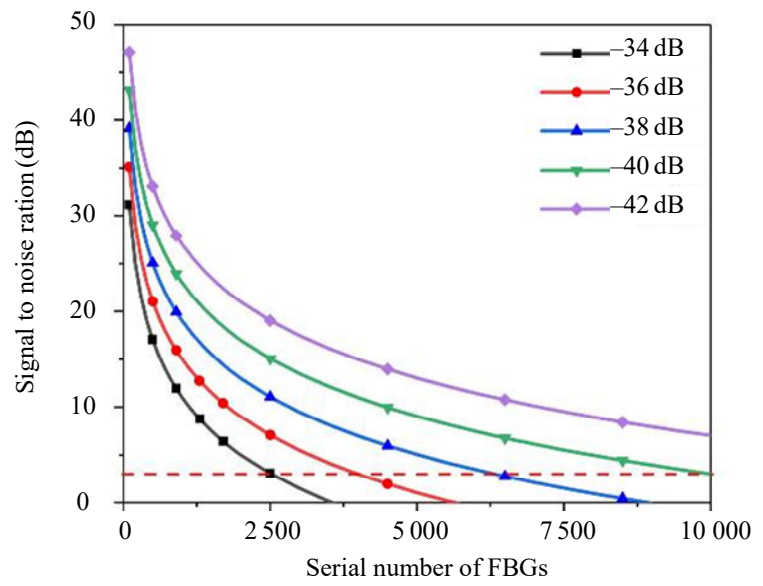

(b)

Fig. 2 Simulation of the reflected power (a) and SNR (b) with the serial number of FBGs owned different reflectivities.

Due to the detection limit of the interrogator, the reflectivity of ultra-weak FBGs cannot be ultimately decreased. As a result, it is necessary to use other ways to improve the signal power and SNR in the FBG array. Usually, the spectral shadowing and multiple-reflection only occur among identical gratings. Increasing the number of central wavelengths of inscribed gratings can reduce the signal crosstalk, so as to improve the multiplexing capacity of the array. For grating interrogator using wavelength scanning time-division multiplexing (WSTDM) technique, the light source has a certain bandwidth, which can be used to measure gratings of several wavelength types. Figure 3 shows the path diagram of the reflected signal in the multi-wavelength FBGs array. The central wavelengths of FBG 1-1, FBG 2-1, and FBG 3-1 are independent, so no signal crosstalk exists in between adjacent gratings. The central wavelengths of FBGs 1-2, 2-2 and 3-2 are consistent with the FBGs 1-1, 2-1, and 3-1, respectively.

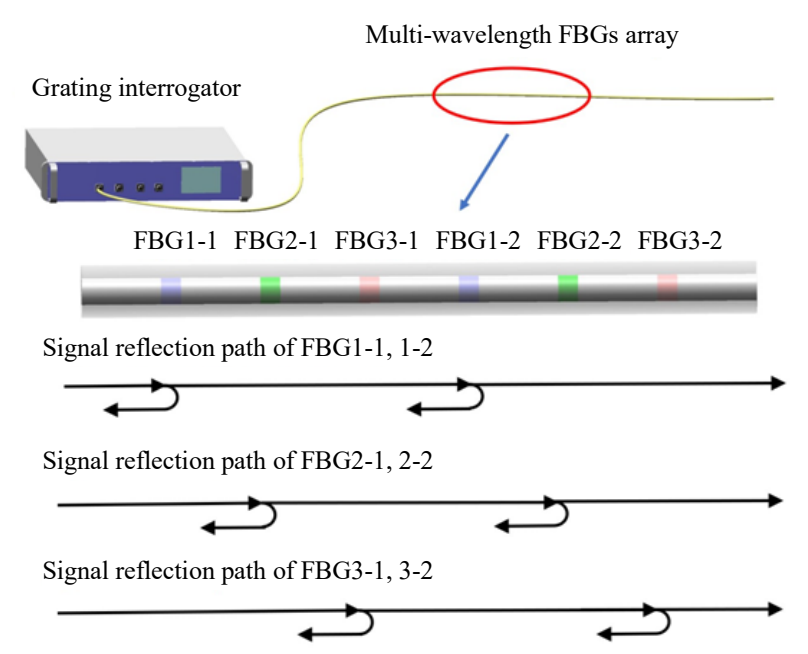

Fig. 3 Path diagram of reflected signal in the multi-wavelength FBGs array.

Based on this model, we explore the relationship between the multiplexing capacity and the number of central wavelengths used in an FBG array. Keeping the reflectivity of a single grating as $-40 \mathrm{~dB}$, the multiplexing capacity of FBGs can be larger, when the number of utilized wavelengths increases from one to four, as plotted in Fig.4(a). The number of recognized gratings can therefore reach 7800 , 12000,14500 , and 16200, respectively. The SNRs of the signal reflected from the corresponding FBGs are shown in Fig.4(b). For the 10 000th grating, the SNR is improved from $3 \mathrm{~dB}$ to $15 \mathrm{~dB}$, which proves that the signal crosstalk is weakened. To summarize, for fabricating large-serial FBGs, decreasing the 
reflectivity of a single grating, and increasing the number of central wavelengths are effective methods to improve the multiplexing capacity. In the following sections, we then focus on the fabrication of two kinds of large-capacity ultra-weak FBG arrays and compare the influence from the introduction of multi-wavelength in the FBG arrays.

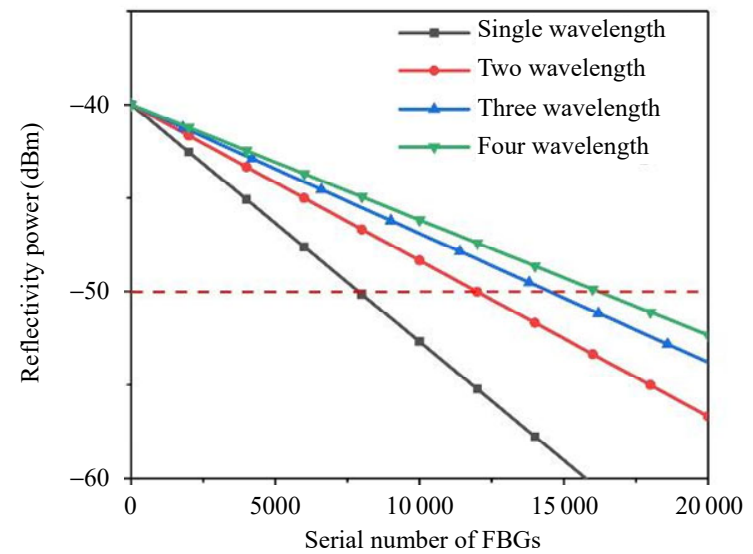

(a)

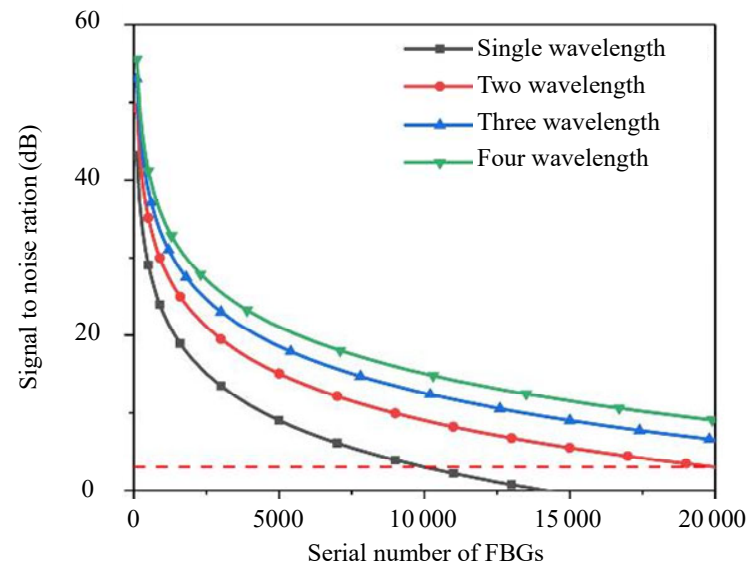

(b)

Fig. 4 Calculations of the power (a) and the SNR (b) with the number of wavelengths used for the grating inscription.

\section{Fabrication and characterization of the on-line written FBG arrays}

The schematic diagram of the on-line fabrication system for FBG arrays is depicted in Fig. 5(a). A fiber preform with a photo-sensitive glass core is fed into a graphite resistance furnace to be heated up to $2000^{\circ} \mathrm{C}$ and drawn to a bare fiber. Right after being pulled out of the furnace, the bare fiber is inscribed by a grating-written platform using a phase mask.
The fiber with written gratings is then coated and cured two times. Using this on-line written system, mass production of large-capacity, high uniformity, and long-distance FBG arrays can be efficiently acquired. Figure 5(b) illustrates the principle of the on-line grating-written platform. Keeping $\sim 0.5 \mathrm{~mm}$ in front of the bare fiber, a phase mask with a size of $10 \mathrm{~mm} \times 10 \mathrm{~mm}$ is mounted. A $248 \mathrm{~nm}$ excimer laser emits pulses of $10 \mathrm{~ns}$ pulse-width and the pulse energy is $10 \mathrm{~mJ}$. The waist profile of the laser beam is $6 \mathrm{~mm} \times 12 \mathrm{~mm}$ and the maximum repetition rate is $500 \mathrm{~Hz}$. The laser beam is narrowed by three cylindrical lenses and then focused onto the phase mask with a beam size of $0.7 \mathrm{~mm} \times 10 \mathrm{~mm}$. When the laser beam passes through the phase mask, \pm 1 st interference fringes are induced, and gratings can then be inscribed on the exposed bare fiber. The period of interference fringe $\Lambda$ (grating pitch) is determined by the following equation [23]:

$$
\Lambda=\Lambda_{\mathrm{PM}} /|p-n|
$$

where $\Lambda_{\mathrm{PM}}$ is the period of phase mask and $p$ and $n$ represent the order of the interference beams. Based on (5), the central wavelength of the inscribed FBG can be calculated:

$$
\lambda_{\mathrm{FBG}}=2 n_{\text {eff }} \Lambda
$$

where $n_{\text {eff }}$ is the effective refractive index of the propagation mode, approximately equal to that of the fiber core.

Keeping the drawing conditions stable, gratings with high uniformity can be inscribed by each laser pulse. The pulse energy of excimer laser and the period of phase mask can be tuned flexibly according to the requirement of gratings. In this study, in order to acquire ultra-weak FBGs of $-40 \mathrm{~dB}$ reflectivity, the pulse energy was set to $\sim 4.5 \mathrm{~mJ}$. To write multi-wavelength FBGs, we use a set of phase masks, as depicted in Fig. 5(c). In the modified setup, three phase masks are mounted on a displacement platform with periods of $1071.93 \mathrm{~nm}$, $1073.31 \mathrm{~nm}$, and $1074.69 \mathrm{~nm}$, respectively. The modified device can control the phase masks to 
move accordingly in front of the bare fiber for writing grating at a certain wavelength,

(a)

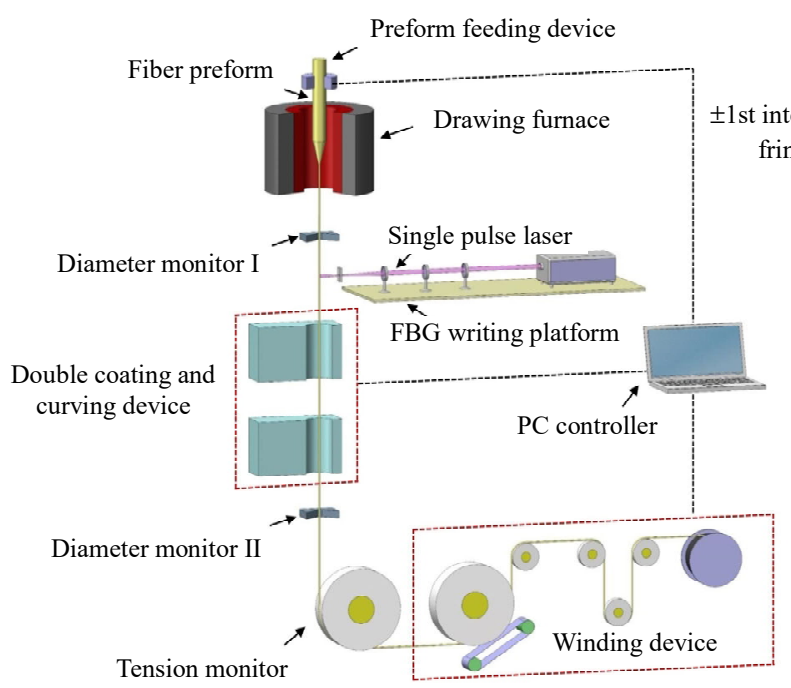

synchronized with the emission of laser pulses at a certain repetition rate.

(b)

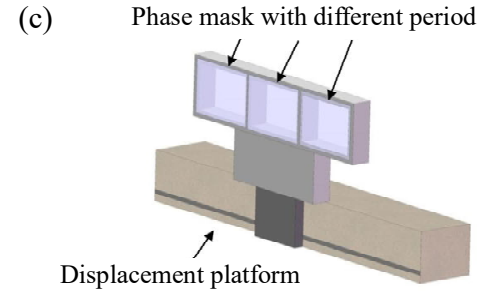

Fig. 5 Schematic diagrams of on-line writing ultra-weak FBGs: (a) optical fiber drawing tower, (b) the grating writing platform, and (c) multi-wavelengths FBG switching device.

Two 10-kilometer-long ultra-weak FBG arrays with an equal grating spacing of $1 \mathrm{~m}$ are fabricated. These FBGs are written one by one in a row, without re-splicing fibers between adjacent gratings. One FBG array uses single-wavelength writing and the other one uses three-wavelength. For these fabricated gratings, the length is $\sim 10 \mathrm{~mm}$ and the reflectivity is $\sim 40 \mathrm{~dB}$. The fabricated FBG arrays are then demodulated using a grating interrogator (LGI-100B, Sentek Instrument, USA), which adopts the WSTDM technique [18]. The central wavelength of the single-wavelength FBG array is plotted in Fig. 6(a). For a total of 10000 FBGs, the majority of central wavelength fluctuates within $\pm 0.19 \mathrm{~nm}$ span, ranging from $1550.44 \mathrm{~nm}$ to $1550.82 \mathrm{~nm}$. The central wavelength of a few gratings runs out of this range, probably induced by the inhomogeneous built-in stress inside the fiber material when coiling the fiber. The small fluctuation in wavelengths indicates that the drawing tower (e.g., preform feeding, fiber drawing, and tension) and the grating writing platform were working stably. Figure 6(b) shows the spectra of 11 selected gratings situated from $1 \mathrm{~m}$ to $10000 \mathrm{~m}$ with an interval of $1000 \mathrm{~m}$.
The reflectivity of each inscribed grating is about $-40 \mathrm{~dB}$, and all gratings can be demodulated by the grating interrogator. The spectra of the first several gratings are very close to central wavelengths at $1550.6 \mathrm{~nm}$. However, the spectra from gratings of the 2 nd half have a certain degree of distortion, induced by the spectral shadowing and multiple-reflection, as discussed before. Due to ultra-weak reflectivity ( $\leq-40 \mathrm{~dB}$ ), such crosstalk can be alleviated so that the reflected signals at the 2nd half are with good strength to be demodulated by the interrogator. Of course, the accuracy of the downstream grating used for sensing units will decline in the practical application. Figure 7(a) plots the central wavelengths of the three-wavelength FBG array. The majority of central wavelengths in this array are about $1550.9 \mathrm{~nm}, 1552.9 \mathrm{~nm}$, and $1554.8 \mathrm{~nm}$. Compared to the single-wavelength FBG array, the fluctuation range of the three-wavelength is increased to $0.55 \mathrm{~nm}$, which is probably induced by the vibration of the continuous movement of the holder of three phase masks. Spectra from 11 gratings are selected from each wavelength, as plotted in Figs. 7(b) to 7(d). All 
grating can be clearly recognized by the demodulator, and the spectra from the 2 nd half have only tiny distortion, indicating lower crosstalk and better performance than those of the FGB array using single-wavelength writing.

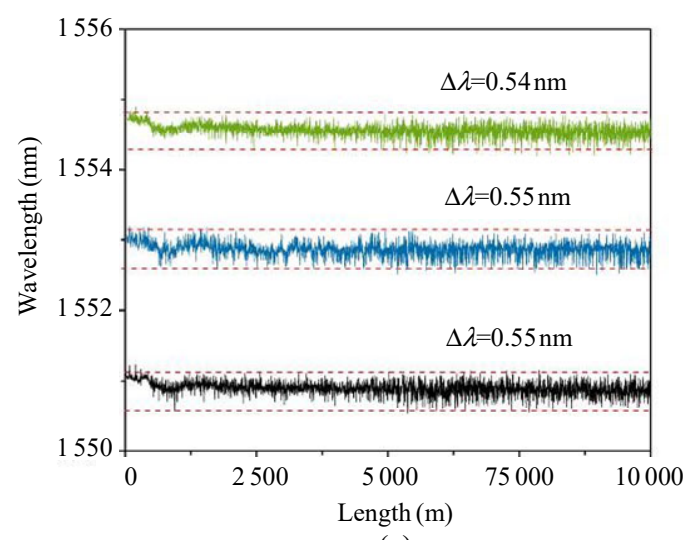

(a)

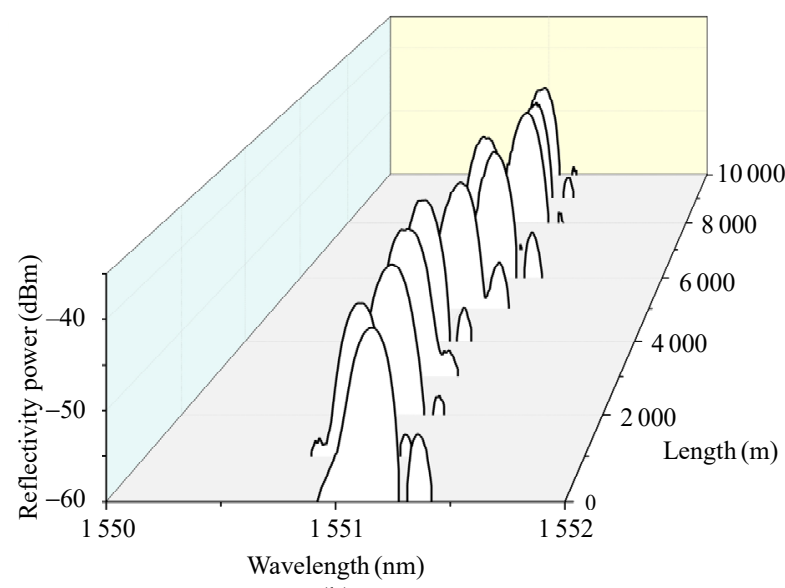

(b)

Fig. 6 Reflective spectra in the single-wavelength array: (a) the consistency of central wavelength of all the 10000 gratings and (b) the spectra of selected gratings situated from $1 \mathrm{~m}$ to $10000 \mathrm{~m}$ with an interval of $1000 \mathrm{~m}$.

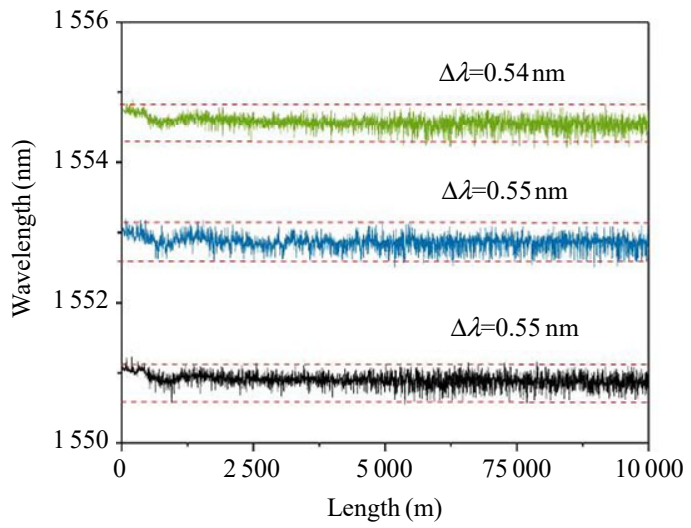

(a)

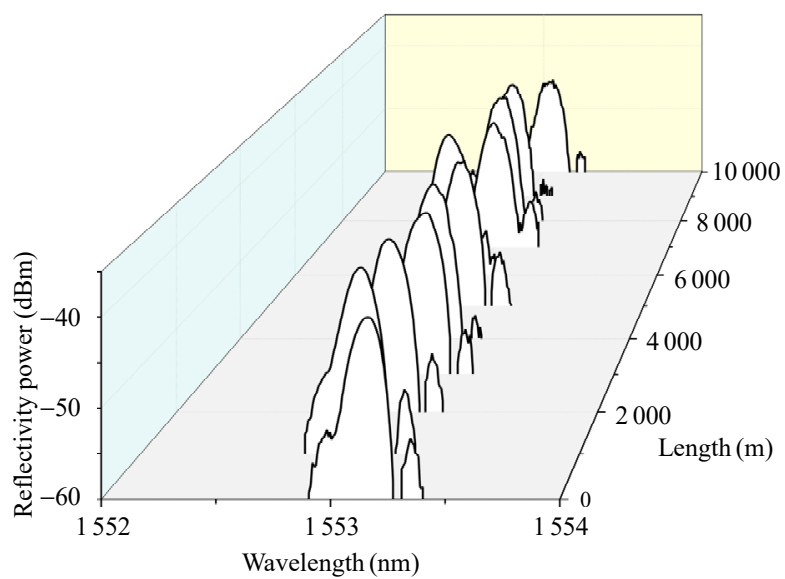

(c)

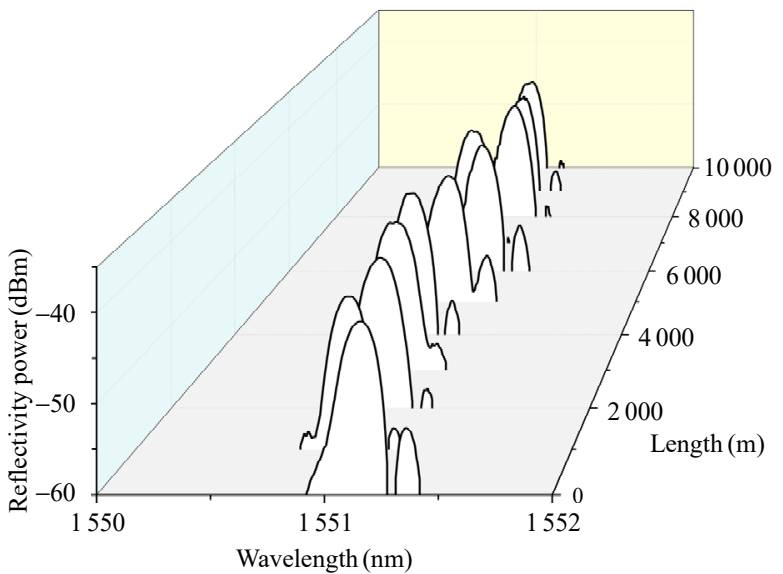

(b)

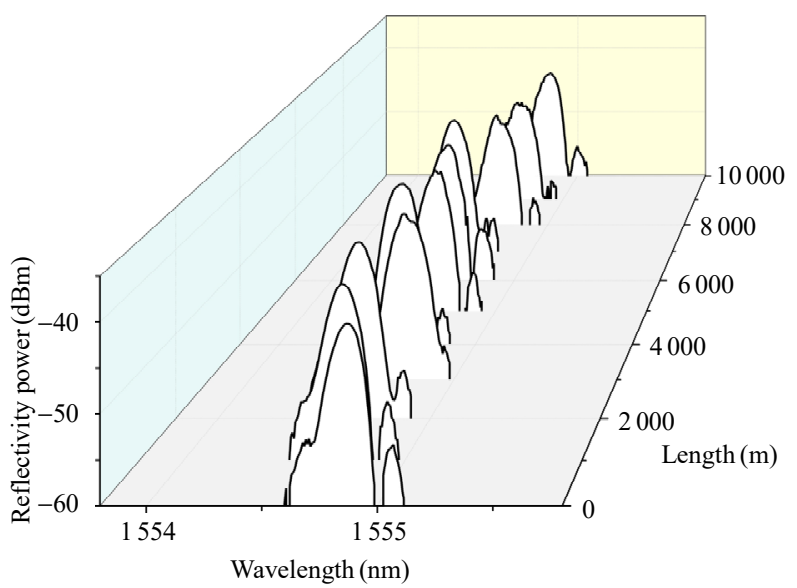

(d)

Fig. 7 Reflective spectra in the three-wavelength array: (a) the consistency of central wavelength of all the 10000 gratings and the spectra of selected gratings with the central wavelength at (b) $1550.9 \mathrm{~nm}$, (c) $1552.9 \mathrm{~nm}$, and (d) $1554.8 \mathrm{~nm}$. 
Figure 8(a) plots the reflected power from 51 single-wavelength gratings over the $10 \mathrm{~km}$ fiber, chosen at an interval of $200 \mathrm{~m}$. With an increase in the number of upstream gratings, the signal power reflected from the downstream grating will decreases linearly, with an attenuation of $0.938 \mathrm{~dB}$ per 1000 gratings. The spectra of the first grating and the last grating are plotted in Fig. 8(b). In comparison, the power of the last grating is reduced by $10.69 \mathrm{~dB}$, and the spectrum is partially distorted. For the three-wavelength FBG array, the attenuation

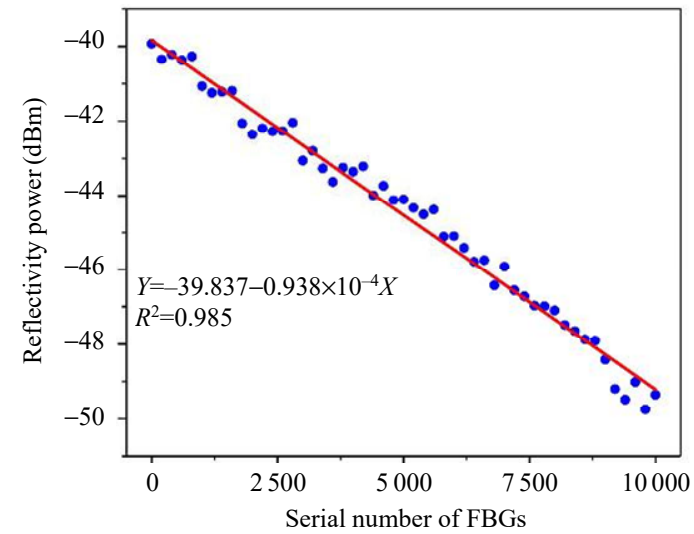

(a)

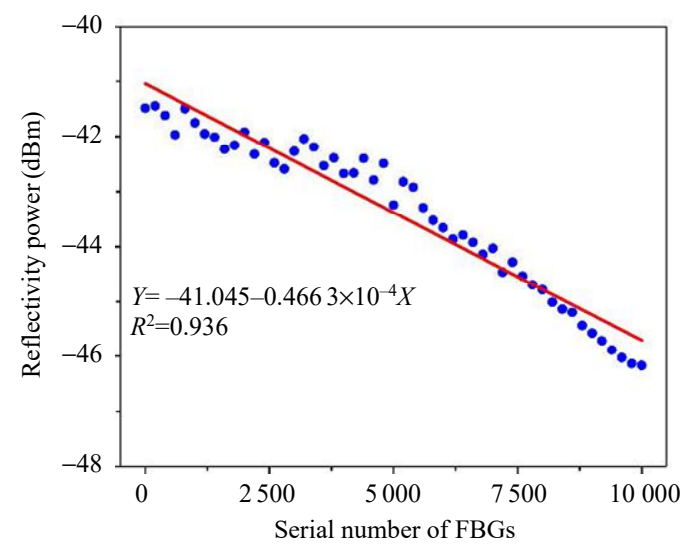

(c) of signal power is reduced to $0.466 \mathrm{~dB}$ per 1000 gratings, as shown in Fig. 8(c). The reduced attenuation indicates that the multiplexing capacity of such a three-wavelength FBG array can be at least doubled, as compared to that in a single-wavelength FBG array. Figure 8(d) plots the spectra of the first and the last gratings of the three-wavelength FBG array. The difference of the power of the central wavelength is reduced to $5.54 \mathrm{~dB}$, and the spectral profile has less distortion, compared to that in the signal-wavelength FBG array.

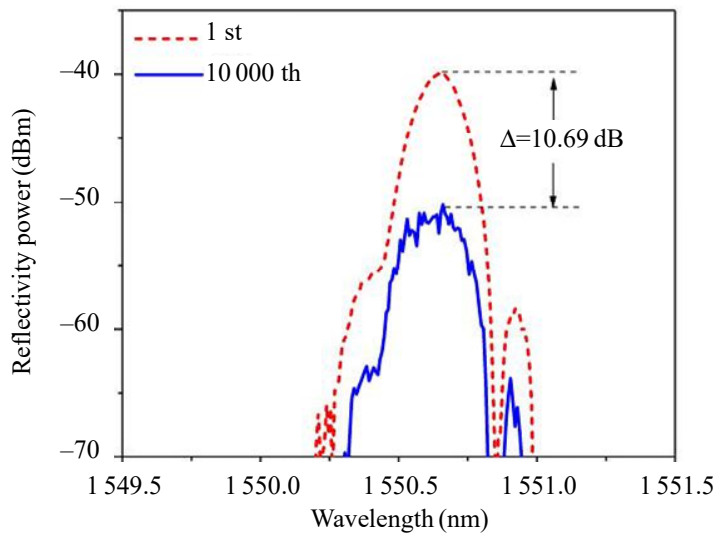

(b)

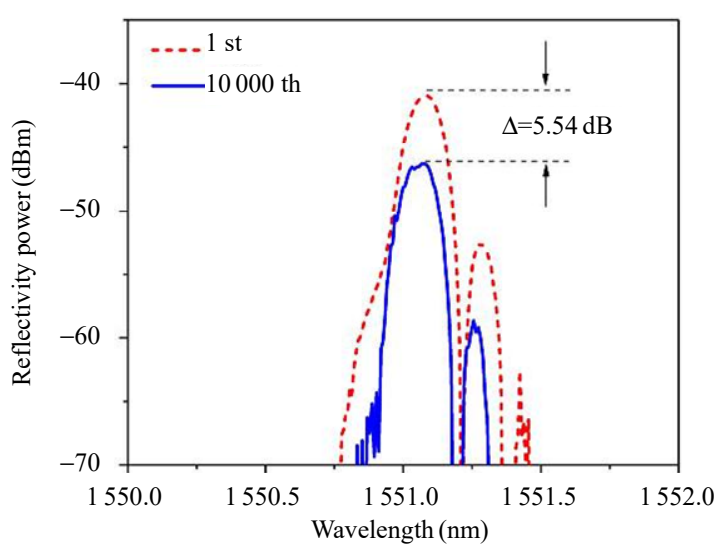

(d)

Fig. 8 Change of reflectivity power of the gratings with increase in serial number in the (a) single wavelength and (c) three wavelengths FBG array. The difference between the first and last gratings in the (b) single wavelength and (d) three wavelengths FBG array.

The transmission loss of two arrays at $1550 \mathrm{~nm}$ and the intensity of ghost grating are measured using an optical time domain reflectometer (OTDR) (AQ7260, Yokogawa, Japan), as re-plotted in Fig. 9. The loss of a conventional single-mode fiber with no grating is $\sim 0.2 \mathrm{~dB} / \mathrm{km}$, while the 10 -kilometer-long
FBG array with 10000 gratings only has a slightly increased attenuation of $\sim 0.48 \mathrm{~dB} / \mathrm{km}$. The power of the actual FBGs and the ghost gratings has obvious differences due to the different reflectivities of these gratings. Usually, the reflectivity of actual FBG is high and stable, while the reflectivity of ghost 
grating is low and decreases with an increase in the distance. For a single-wavelength FBG array, the power difference between the end grating and the first ghost grating is $5.62 \mathrm{~dB}$, which can be clearly distinguished by the system. The power difference in the three-wavelength FBG array is increased to $8.14 \mathrm{~dB}$, indicating an advanced feature of robust signal against crosstalk. Based on the above studies, it is clear that using multi-wavelength and ultra-weak FBGs, the systems possess better accuracy and greater multiplexing capacity for building large-scale sensing networks.

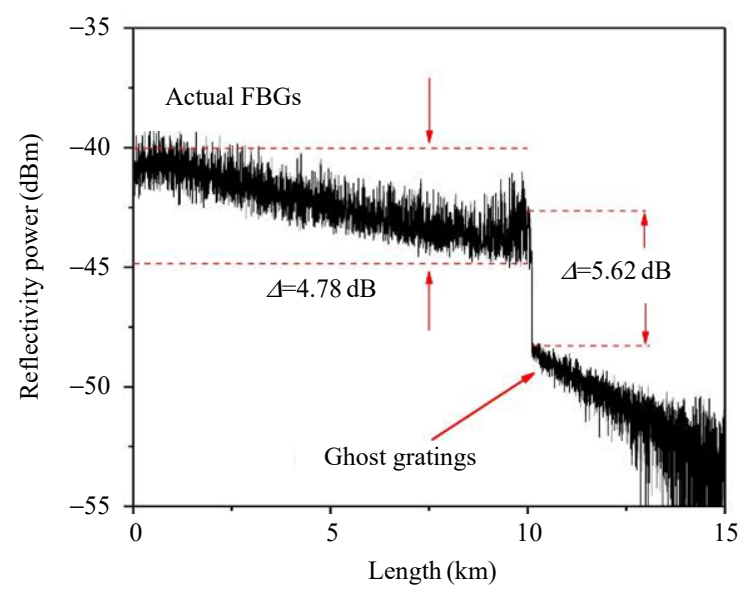

(a)

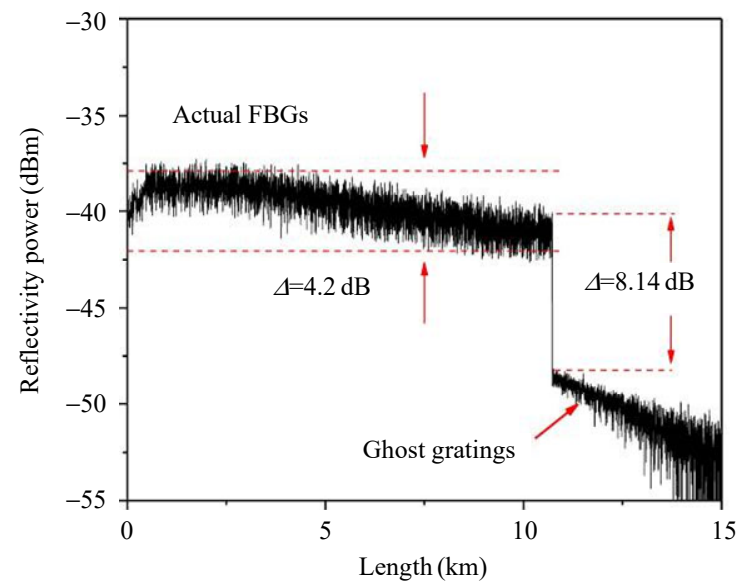

(b)

Fig. 9 Transmission loss of the (a) single wavelength and (b) three wavelengths array gathered by an OTDR.

\section{Conclusions}

In this paper, two main factors influencing the quality of signals reflected from gratings are studied for large-scale FBG arrays. In the reflective index range of $34 \mathrm{~dB}-42 \mathrm{~dB}$, reducing the reflectivity of a single grating and increasing the number of central wavelengths can effectively eliminate the influences from the spectral shadowing and multiple-reflection induced crosstalk. The experimental results show that ultra-weak FBGs written by a single-wavelength can realize the application of 10-kilometer-long sensing networks with the number of units of 10000 . However, it is possible to double this figure by using a three-wavelength FBG array. Such a multi-wavelength FBG array has promising potentials in many aspects regarding large-scale safety production or structural surveillance.

\section{Acknowledgment}

All authors thank the National Engineering Laboratory for Fiber Optic Sensing Technology for offering the experimental equipment. This work was supported by the National Natural Science Foundation of China (Grant No. 61290311); Hubei Key Laboratory of Radiation Chemistry and Functional Materials (Grant No. 2019-20KZ08); State Key Laboratory of Advanced Technology for Materials Synthesis and Processing (Grant No. 2019-KF-11).

Open Access This article is distributed under the terms of the Creative Commons Attribution 4.0 International License (http://creativecommons.org/licenses/by/4.0/), which permits unrestricted use, distribution, and reproduction in any medium, provided you give appropriate credit to the original author(s) and the source, provide a link to the Creative Commons license, and indicate if changes were made.

\section{References}

[1] A. Ukil, H. Braendle, and P. Krippner, distributed temperature sensing: review of technology and applications," IEEE Sensors Journal, 2011, 12(5): 885-892.

[2] V. F. Bense, T. Read, O. Bour, T. Le Borgne, T. Coleman, S. Krause, et al., "Distributed temperature sensing as a downhole tool in hydrogeology," Water Resources Research, 2016, 30: 1017-1035. 
[3] U. J. Dreyer, F. Mezzadri, G. Dutra, T. da Silva, C. A. Bavastri, E. Vagner, et al., "Quasi-distributed optical fiber transducer for simultaneous temperature and vibration sensing in high-power generators," IEEE Sensors Journal, 2018, 18(4): 1547-1554.

[4] H. Guo, G. Xiao, N. Mrad, and J. Yao, "Fiber optic sensors for structural health monitoring of air platforms," Sensors, 2011, 11(4): 3687-3705.

[5] X. Liu, Q. Sun, J. Wo, M. Zhang, and D. Liu, "Hybrid TDM/WDM-based fiber-optic sensor network for perimeter intrusion detection," Journal of Lightwave Technology, 2012, 30(8): 1113-1120.

[6] B. Lee, "Review of the present status of optical fiber sensors," Optical Fiber Technology, 2003, 9(2): 57-79.

[7] M. K. Saxena, S. D. Raju, R. Arya, S. V. G. Ravindranath, S. Kher, and S. M. Oak, "Optical fiber distributed temperature sensor using short term Fourier transform based simplified signal processing of Raman signals," Measurement, 2014, 47: 345-355.

[8] L. Zhang, Z. Wang, J. Li, J. Zeng, Y. Li, X. Jia, et al., "Ultra-long dual-sideband BOTDA with balanced detection," Optics and Laser Technology, 2015, 68: 206-210.

[9] J. Jiang, J. Xiong, Z. Wang, Z. Wang, Z. Qiu, C. Liu, et al., "Quasi-distributed fiber-optic acoustic sensing with MIMO technology," IEEE Internet of Things Journal, 2021, 8: 1.

[10] Z. Wang, J. Jiang, J. Xiong, J. Xiong, Z. Qiu, C. Liu, et al., "Quasi-distributed acoustic sensing with interleaved identical chirped pulses for multiplying the measurement slew-rate," Optics Express, 2020, 28(26): 38465-38479.

[11] G. Laffont, R. Cotillard, and P. Ferdinand, "Multiplexed regenerated fiber Bragg gratings for high-temperature measurement," Measurement Science and Technology, 2013, 24(9): 094010.

[12] D. Kinet, M. Patrice, K. W. Goossen, L. Qiu, D. Heider, and C. Caucheteur, "Fiber Bragg grating sensors toward structural health monitoring in composite materials: challenges and solutions," Sensors, 2014, 14(4): 7394-7419.

[13] Y. Wang, J. Gong, D. Y. Wang, B. Dong, W. Bi, and A. Wang, "A quasi-distributed sensing network with time-division-multiplexed fiber Bragg gratings,"
IEEE Photonics Technology Letters, 2010, 23(2): 70-72.

[14] D. J. F. Cooper, T. Coroy, and P. W. E. Smith, "Time-division multiplexing of large serial fiber-optic Bragg grating sensor arrays," Applied Optics, 2001, 40(16): 2643-2654.

[15] Y. Dai, Y. Liu, J. Leng, G Deng, and A. Asundi, “A novel time-division multiplexing fiber Bragg grating sensor interrogator for structural health monitoring," Optics and Lasers in Engineering, 2009, 47(10): 1028-1033.

[16] H. Guo, L. Qian, C. Zhou, Z. Zheng, Y. Yuan, R. Xu, et al., "Crosstalk and ghost gratings in a large-scale weak fiber Bragg grating array," Journal of Lightwave Technology, 2017, 35(10): 2032-2036.

[17] W. Wang, J. Gong, B. Dong, D. Y. Wang, T. J. Shillig, and A. Wang, "A large serial time-division multiplexed fiber Bragg grating sensor network," Journal of Lightwave Technology, 2012, 30(17): 2751-2756.

[18] Z. Luo, H. Wen, H. Guo, and M. Yang, "A time- and wavelength-division multiplexing sensor network with ultra-weak fiber Bragg gratings," Optics Express, 2013, 21(19): 22799-22807.

[19] C. G. Askins, M. A. Putnam, H. J. Patrick, and E. J. Friebele, "Fibre strength unaffected by on-line writing of single-pulse Bragg gratings," Electronics Letters, 1997, 33(15): 1333-1334.

[20] L. Ma, C. Ma, Y. Wang, D. Y. Wang, and A. Wang, "High-speed distributed sensing based on ultra weak FBGs and chromatic dispersion," IEEE Photonics Technology Letters, 2016, 28(12): 1344-1347.

[21] H. Guo, J. Tang, X. Li, Y. Zheng, H. Yu, and H. Yu, "On-line writing identical and weak fiber Bragg grating arrays," Chinese Optics Letters, 2013, 11(3): 030602 .

[22] H. Guo, F. Liu, Y. Yuan, H. Yu, and M. Yang, "Ultra-weak FBG and its refractive index distribution in the drawing optical fiber," Optics Express, 2015, 23(4): 4829-4838.

[23] Y. Zheng, H. Yu, H. Guo, X. Li, and D. Jiang, "Analysis of the spectrum distortions of weak fiber Bragg gratings fabricated in-line on a draw tower by the phase mask technique," Journal of Lightwave Technology, 2014, 33(12): 2670-2673. 